\title{
THE CLAIRVOYANT DEMON HAS A HARD TASK
}

\author{
PETER GÁCS
}

\begin{abstract}
Consider the integer lattice $L=\mathbb{Z}^{2}$. For some $m \geq 4$, let us color each column of this lattice independently and uniformly into one of $m$ colors. We do the same for the rows, independently from the columns. A point of $L$ will be called blocked if its row and column have the same color. We say that this random configuration percolates if there is a path in $L$ starting at the origin, consisting of rightward and upward unit steps, and avoiding the blocked points. As a problem arising in distributed computing, it has been conjectured that for $m \geq 4$, the configuration percolates with positive probability. This has now been proved (in a later paper) for large $m$. We prove that the probability that there is percolation to distance $n$ but not to infinity is not exponentially small in $n$. This narrows the range of methods available for proving the conjecture.
\end{abstract}

\section{Statement of the Result}

1.1. Introduction. Let $x=(x(0), x(1), \ldots)$ be an infinite sequence and $u=(u(0), u(1), \ldots)$ be a binary sequence with elements in $\{0,1\}$. Let $s_{n}=\sum_{i=0}^{n-1} u(i)$. We define the delayed version $x^{(u)}$ of $x$, by

$$
x^{(u)}(n)=x\left(s_{n}\right) .
$$

Thus, if $i=s_{n}$ then $x^{(u)}(n)=x(i)$, and $x^{(u)}(n+1)=x(i)$ or $x(i+1)$ depending on whether $u(n)=0$ or 1 . If $u(n)=0$ then we can say that $x^{(u)}$ is delayed at time $n+1$. For two infinite sequences $x, y$ we say that they do not collide if there is a delay sequence $u$ such that for each $n$ we have

$$
x^{(u)}(n) \neq y^{(1-u)}(n) .
$$

Here, $1-u$ is the delay sequence complementary to $u$ : thus, $y^{(1-u)}$ is delayed at time $n$ if and only if $x^{(u)}$ is not.

For a given $m>1$, suppose that $X=(X(0), X(1), \ldots)$ is an infinite sequence of independent random variables, and $Y=(Y(0), Y(1), \ldots)$ is another such sequence, also independent of $X$, where all variables are uniformly distributed over $\{0, \ldots, m-1\}$. The following theorem has been conjectured in [2].

Proposition 1.1 (See [3]). If $m$ is sufficiently large then with positive probability, $X$ does not collide with $Y$.

The sequences $X, Y$ can be viewed as two independent random walks on the complete graph $K_{m}$, and then the problem is whether a "clairvoyant demon", i.e. a being who knows in advance both infinite sequences $X$ and $Y$, can introduce delays into these walks in such a way that they never collide.

1.2. Graph reformulation. We define a graph $G=(V, E)$ as follows. $V=\mathbb{Z}_{>0}^{2}$ is the set of points $(i, j)$ where $i, j$ are positive integers. Let us define the distance of two points $(i, j),(k, l)$ as $|k-i|+|l-j|$ ( $L_{1}$ distance). When representing the set $V$ of points $(i, j)$ graphically, the right direction is the one of growing $i$, and the upward direction is the one

Key words and phrases. Dependent percolation, scheduling, distributed computing. 
of growing $j$. The set $E$ of edges consists of all pairs of the form $((i, j),(i+1, j))$ and $((i, j),(i, j+1))$.

Given $X, Y$ as in the theorem, let us say that a point $(i, j)$ has color $k$ if $X(i)=Y(j)=k$. Otherwise, it has color -1 , which we will call white. It is easy to see that $X$ and $Y$ do not collide if and only if there is an infinite directed path in $G$ starting from $(0,0)$ and proceeding on white points. Indeed, each path corresponds to a delay sequence $u$ such that $u(n)=1$ if and only if the edge is horizontal. Thus, the two sequences do not collide if and only if the graph of white points "percolates". We will say that there is percolation if the probability that there is an infinite path is positive.

It has been shown independently in [1] and [4 that if the graph is undirected then there is percolation even for $m=4$. In traditional percolation theory, when there is percolation then typically (unless the probability of blocking is at a "critical point") the probability that there is percolation to a distance $n$ but no percolation to infinity is exponentially small in $n$. This is the case also in the papers cited above, but it is not true for the directed percolation problem we are facing.

Theorem 1. If there is percolation from the origin to infinity with positive probability then the probability of percolating from the origin to distance $n$ but not to infinity is at least $C n^{-\alpha}$ for some constants $C, \alpha>0$ depending only on $m$.

\section{THE PROOF}

Let $b_{m}=(0,1,2, \ldots, m-1)$ be called the basic color sequence of length $m$ : it is simply the list of all different colors. Let $b_{m}^{\prime}$ be the reverse of $b_{m}$, i.e. $b_{m}^{\prime}(i)=b_{m}(m-i-1)$. Let $\mathcal{E}_{n, k}$ be the event that for all $i \in[0, k-1], j \in[0, m-1]$ we have

$$
Y(n+i m+j-1)=b_{m}^{\prime}(j),
$$

i.e. starting with the index $n-1$, the sequence $Y$ has $k$ consecutive repetitions of $b_{m}^{\prime}$. We say that $i$ is an index of the occurrence of $b_{m}$ in the sequence $X$ if $X(i+j)=b_{m}(j)$ for $j \in[0, m-1]$. For $i>0$, let $\tau_{i}$ be the $i$-th index of occurrence of $b_{m}$ in $X$. Let $\mathcal{F}_{n, k}$ be the event that for all $i \in[1, n]$ we have

$$
\tau_{i+1}-m-\tau_{i} \leq k-1
$$

and also $\tau_{1} \leq k-1$.

Lemma 2.1. If for some integer $n>0$ both $\mathcal{E}_{n, k}$ and $\mathcal{F}_{n, k}$ hold then there is no white infinite directed path.

Proof. Let us assume that there is a white infinite path. Since there are $n$ consecutive copies of $b_{m}$ in $X$, the $i$-th one starting at index $\tau_{i}$, for each $p \in[1, n]$ there must be a vertical step in the path with an $x$ projection in $\left[\tau_{p}, \tau_{p}+m-1\right]$. Therefore the path ascends to the segment $\left[0, \tau_{n}+m-1\right] \times\{n-1\}$, before its $x$ projection reaches $t_{n}$.

For each $1 \leq p \leq n, 0 \leq q<k$ there is a diagonally descending sequence of $m$ colored points

$$
\left\{\left(\tau_{p}+j, n+(q+1) m-j-2\right): j \in[0, m-1]\right\} .
$$

For a fixed $p$ there are $k$ such diagonal barriers stacked above each other, forming an impenetrable column of height $\mathrm{km}$. The path would have to ascend between two of these columns, say between column $i$ and $i+1$. The distance of two consecutive columns from each other is

$$
\tau_{i+1}-m-\tau_{i} \leq k-1
$$


Since there are $k$ consecutive copies of $b_{m}^{\prime}$ in $Y$ starting at index $n-1$, for each $q \in[0, k-1]$ there must be a horizontal step in the path with a height in $n+q m-1+[0, m-1]$. Since the distance of the two columns is at most $k-1$, it is not possible for the path to pass between the two columns. (The same holds for the space before the first column.)

Lemma 2.2. There is a constant $\alpha$ such that for all $s>0$, there is a $k$ with

$$
\operatorname{Prob}\left(\mathcal{E}_{n, k}\right) \geq m^{-m} n^{-\alpha(s+1)}, \quad \operatorname{Prob}\left(\mathcal{F}_{n, k}\right) \geq 1-n^{-s} .
$$

Proof. With $p_{1}=m^{-m}$, we have $\operatorname{Prob}\left(\mathcal{E}_{n, k}\right)=p_{1}^{k}$. Let us estimate the probability of $\mathcal{F}_{n, k}$. The probability that $\tau_{1}>k-1$ is upperbounded by the probability that a copy of $b_{m}$ does not begin at $i$ in $X$ for any $i$ in $\{0, m, \ldots,\lfloor(k-1) / m\rfloor m\}$, which is

$$
\left(1-p_{1}\right)^{\lfloor(k-1) / m\rfloor+1} \leq\left(1-p_{1}\right)^{k / m}<e^{-p_{1} k / m} .
$$

The same estimate holds for the probability of (11) assuming that the similar conditions for smaller $i$ have already been satisfied. Hence

$$
\operatorname{Prob}\left(\mathcal{F}_{n, k}\right)>1-n e^{-p_{1} k / m} \text {. }
$$

Let us choose

$$
k=\left\lceil(s+1)(m \log n) / p_{1}\right\rceil
$$

for some $s>0$, then $\operatorname{Prob}\left(\mathcal{F}_{n, k}\right)>1-n^{-s}$, while

$$
\operatorname{Prob}\left(\mathcal{E}_{n, k}\right) \geq p_{1} n^{\frac{m \log p_{1}}{p_{1}}(s+1)} .
$$

Proof of Theorem 1. Assume that there is an infinite path with some positive probability $P_{0}$. Choose $s$ such that $n^{-s}<0.5 P_{0}$ and choose $k$ as a function of $s$ as in (2). Then the probability that $\mathcal{F}_{n, k}$ holds and there is an infinite path is at least $0.5 P_{0}$. Let $\mathcal{G}_{n}$ be the event that there is a path leaving the rectangle $\left[0, \tau_{n}\right] \times[0, n-1]$. Then $\operatorname{Prob}\left(\mathcal{F}_{n, k} \wedge \mathcal{G}_{n}\right) \geq 0.5 P_{0}$. Since $\mathcal{F}_{n, k} \wedge \mathcal{G}_{n}$ is independent of $\mathcal{E}_{n, k}$, we have

$$
\operatorname{Prob}\left(\mathcal{E}_{n, k} \wedge \mathcal{F}_{n, k} \wedge \mathcal{G}_{n}\right) \geq 0.5 P_{0} m^{-m} n^{-\alpha(s+1)} .
$$

The author is thankful to János Komlós and Endre Szemerédi for telling him about the problem.

\section{REFERENCES}

[1] P.N. Balister, B. Bollobas, and A.N. Stacey, Dependent percolation in two dimensions, Tech. report, 1999.

[2] D. Coppersmith, P. Tetali, and P. Winkler, Collisions among random walks on a graph, SIAM J. Discrete Math 6 (1993), no. 3, 363-374.

[3] Peter Gács. Clairvoyant scheduling of random walks. math.PR/0109152

[4] P. Winkler, Dependent percolation and colliding random walks, Tech. report, 1999.

Computer Science Department, Boston University

E-mail address: gacs@bu.edu 